\title{
Luces y sombras de la historia de la renta italiana ${ }^{1}$
}

\author{
SiMONE Misiani \\ Universidad de Téramo \\ smisiani@unite.it
}

Recibido: 01.02.2010

Aceptado: 17.05 .2010

\section{LA ESTADÍSTICA PÚBLICA Y EL CENSO DE 1937-1939}

Los acontecimientos de los últimos años, después del 11 de septiembre de 2001, han evidenciado la distancia entre la imagen y la realidad de hechos y personajes cruciales. La crisis más reciente de las finanzas públicas ha desvelado, una vez más, el problema de la falsificación de las cifras y la necesidad de dotar a las democracias de instituciones capaces de garantizar una información independiente. Este problema se vuelve más urgente a medida que aumenta la implicación de los individuos en decisiones colectivas. Desde este punto de vista, la historia de la renta nacional ofrece a la investigación una clave para profundizar el estudio de la decisión pública y analizar el funcionamiento del sistema político. Este trabajo tiene como objetivo reconstruir los origenes, en Italia, de un sistema de contabilidad nacional en relación con el problema de la decisión política. El concepto de estadística económica es visto no en sentido estático, es decir como dato objetivo adquirido de una vez y para siempre, sino como parte constitutiva de la vida política (Desrosiéres, 1993 y 2000).

La invención de la contabilidad nacional fue un acontecimiento de alcance histórico, es un producto de las democracias industriales y en particular de Estados Unidos. El reconocimiento de la contabilidad nacional se desarrolla como consecuencia de la crisis económica mundial, cuando los gobiernos empiezan a adoptar medidas de programación para salir de la Depresión. Se restablece la confianza en la relación entre libertad individual y felicidad pública que la crisis económica parecía haber partido, cuando los marxistas creían que colectivismo y dirigismo habrían salvado el mundo de las injusticias. Las fechas

\footnotetext{
1 Traducción del italiano: Anna Scicolone.
} 
que marcan un cambio sustancial son 1937 y 1938, con los estudios sobre la elaboración de la renta nacional procedentes de Estados Unidos y Suecia. ${ }^{2}$ Con este paso empezó una revolución que se extendería, durante la guerra fría, a todos los principales gobiernos del mundo democrático. La enseñanza de Colin Clark, economista de Cambridge y autor del volumen The national income 1924-1931 publicado en 1932, tuvo una repercusión fundamental en la elaboración del cálculo de la renta nacional. Clark influenció los estudios dirigidos por Estados Unidos a lo largo de los años y ejerció su magisterio sobre Richard Stone, servidor del Estado en el gabinete inglés de guerra y autor del primer proyecto de esquema de renta nacional, luego adoptado por las Naciones Unidas.

La decisión del gobierno de Estados Unidos, tal como la de otros países europeos, de adoptar la teoría de Keynes estimuló los avances en la investigación probabilística. La recepción de este cambio se vinculaba a la elección política de la programación y a dos adquisiciones de orden científico: el método matemático probabilístico, hecho posible por el estudio muestral, y la tecnología de recuento de datos, culminada con la invención de la informática. Los estadísticos estaban convencidos de poder alcanzar un conocimiento global de la renta nacional y orientar las decisiones públicas. Las primeras tentativas fueron acompañadas por polémicas y críticas que ponían en tela de juicio la posibilidad de un procedimiento basado en encuestas muestrales. Volveremos más adelante sobre este tema, con referencia al caso italiano. Empezó a ser aceptada, aunque con reservas y dudas, la posibilidad del cálculo de la renta nacional y la elaboración de una verdadera contabilidad nacional, concepto tomado en prestamo de la cultura empresarial. El conocimiento cuantitativo de la renta nacional debería poder sustentar las decisiones de la política económica.

Italia, como otros régimenes totalitarios (Alemania y Unión Soviética), no adoptó esta innovación ya que reflejaba una concepción democrática y liberal de la planificación. Sin embargo, en este período se dieron decisivos pasos adelante en el campo del conocimiento teórico, ya antes de la caída del Fascismo. En Italia existía una tradición de la estadística del Resorgimento, gracias a la cual se habían promovido las encuestas sociales en época liberal (Marucco 1996, Baffigi 2007 y Favero 2001 y 2009).

En esas encuestas ya era implícita una función política de la estadística, o sea de contribución a la reducción de la desigualdad social, pero estaba completamente ausente la comparación con cuestiones de naturaleza macroeconómica y financiera. Durante la Gran Guerra se emprendieron los primeros estudios sobre

${ }^{2}$ Los estudios más importantes en el período de anteguerra son los realizados sobre la renta sueca (E. Lindhal, E. Dahlgren, K. Kock, National income of Sweden 1861-1930, London, 1937), y la renta en Estados Unidos (S. Kuznets, National income and capital formation, 1915-1935, Nueva York,1937). Un intento anterior de medir la formación de capital se les debe a los estadísticos alemanes de la república de Weimar al principio de los años treinta. Sobre este tema existe una amplia bibliográfica. Por ejemplo véase, Vanoli, 2005, pp. 18-23. Sobre Estados Unidos, véase, Didier (2009).

EMPIRIA. Revista de Metodología de Ciencias Sociales. N. ${ }^{2}$ 21, enero-junio, 2011, pp. 143-169. ISSN: 1139-5737 
el cálculo de la renta, en función de la defensa del poder de adquisición de las capas populares y se hizo hincapié en la necesidad de una reforma de las instituciones, dependiendo del cambio del sistema productivo. Las oficinas estadísticas de cada ciudad prepararon las intervenciones correspondientes para afrontar fenómenos contingentes, (inflación, aumento del coste de la vida, paro y problema de las viviendas) y sugirieron reformas estructurales (planificación territorial, control público de los servicios municipales, etc.). En este período, emergen jóvenes actores del círculo milanés que se formó en los años veinte en correspondencia con el cambio de las empresas y los problemas derivados por la crisis económica. Me refiero a Raffaele Mattioli, Ezio Vanoni, Libero Lenti, Ferdinando Di Fenizio, Carlo Rosselli, Guglielmo Tagliacarne, Alessandro Molinari y también a Paolo Baffi. Son los artífices de una revolución metodológica que veía, en la primacía de la cultura conoscitiva, el instrumento para orientar una política de reformas estructurales. Son los exponentes de una clase dirigente, formada por gente competente y experta, que acompaña el curso del desarrollo italiano desde los años treinta hasta los sesenta. A partir de un programa municipal, se trabaja para el nacimiento de una estadística nacional en un momento fuertemente influenciado por el cambio del sistema político nacional. En Italia, la adopción de esta tendencia encontró varios obstaculos; en primer lugar, por la incapacidad de las clases dirigentes liberales para introducir elementos de reforma en las instituciones capaces de dar voz a la Italia de las fábricas. En segundo lugar, por la injerencia de la política del Régimen fascista que limitó los espacios de autonomía de la estadística oficial y no reconoció en el crecimiento de la economía el origen y el objetivo último de la decisión pública.

A principios de los años treinta, los instrumentos de encuesta periódica eran inadecuados para desarrollar tareas relacionadas con las transformaciones que estaba viviendo la economía mundial. Italia contaba con los cálculos de la revista Prospettive economiche, dirigida por el estadístico Giorgio Mortara, docente en la Universidad Bocconi de Milán; y enseguida después por importancia, con la revista Movimento commerciale dell'Italia, editada por el Banco Comercial Italiano (Comit). En esa revista el Banco Comercial había publicado hasta 1932 los datos periódicos sobre el movimiento de los precios a nivel mundial. De hecho, la obra fue una contribución fundamental a la metodología del cálculo de la renta nacional. Se planteaba el problema de propocionar al país los instrumentos de encuesta para el cálculo de los indicadores de la riqueza, de la renta nacional y de la individual, a los que tenía que ser añadido otro dato fundamental: el curso del comercio exterior. El cálculo de la contabilidad económica y social encontró una primera apreciación en el ámbito de la reforma económica que seguió a la quiebra de los bancos mixtos, con la creación del Instituto para la Reconstrucción Industrial (IRI) en 1933, el ente de derecho público nacido para socorrer a las empresas afectadas por la Depresión, y con la puesta en marcha de la política de saneamiento del sistema productivo. Uno de los primeros actos de Raffaele Mattioli, en calidad de administrador delegado del Comit, fue la consolidación del departamento estudios. Al frente de la dirección del departamen-

EMPIRIA. Revista de Metodología de Ciencias Sociales. N. ${ }^{2}$ 1, enero-junio, 2011, pp. 143-169. ISSN: $1139-5737$ 
to puso a Antonello Gerbi, que desempeñó esta función hasta el verano de 1938, cuando fue obligado a dejarlo por la promulgación de las leyes raciales (Gerbi, 2002; Rodano,1983). En estos años, en el departamento estudios entraron Ugo La Malfa, Giovanni Malagodi y Guido Carli. En el Comit se estudiaba a Keynes, el laborismo inglés y el New Deal americano. En el mismo período empieza a circular el debate sobre los consumos en Italia. Los grupos industriales más importantes se dotaron de centros de estudios estadísticos y planificación empresarial: Libero Lenti trabajó en la Snia Viscosa, Ferdinando Di Fenizio en la Montecatini, Ferruccio Parri en la Edison, Pasquale Saraceno en el IRI, Guglielmo Tagliacarne en la Cámara de Comercio de Milán y Mario Saibante en la Confindustria. Los intereses de las empresas se hicieron coincidir con los de la colectividad de los italianos; los aumentos de los niveles de productividad y eficiencia empresarial deberían conducir a la mejoría de los niveles de vida y al bienestar social. Se establecieron las premisas de la programación en un contexto de tipo corporativo, condicionado por la falta de una dialéctica conflictiva democrática.

Mussolini acogió, y en cierta medida avaló, el análisis de los estadísticos dirigido a una política de la reducción de las desigualdades aunque, al mismo tiempo, trató de servirse de ella para los objetivos del consentimiento al régimen. En 1935, después de la agresión de Etiopía y las sanciones económicas a Italia, Mussolini hizo público el objetivo de la autarquía, o sea, el logro de la autosuficiencia económica. Reivindicó el mito de la «gran proletaria», capaz de proponer una tercera vía entre capitalismo y comunismo, entre individualismo y colectivismo; era una orientación antiliberal y anticapitalista a la vez. De igual manera Mussolini aceptó la solicitud para consolidar el papel del banco central, como ya ocurría en Gran Bretaña y Estados Unidos, dando espacio a las iniciativas de estudio sobre la renta nacional dirigida a introducir una política económica que solucionara el retraso del desarrollo italiano. El momento decisivo fue la adopción de la ley bancaria en 1936. El instituto de emisión adquirió un poder de dirección sobre la moneda, y además, dentro del programa de consolidación de la línea de Bankitalia, la propuesta de creación de un Servicio de Estudios fue acogida para permitir un contacto directo con la verdadera economía. El proyecto había sido ideado por Giorgio Mortara que logró plasmar en el nuevo organigrama Paolo Baffi, su adjunto a la cátedra en la Universidad Bocconi, y que en la inmediata posguerra se convirtió en el responsable del Servicio (Baffi, 1990, 93-151; Tuccimei, 2005; Caracciolo, 1993; Gigliobianco, 2006, 145-148 y 308313).

En estos meses, dentro del Servicio de Estudios se formó un grupo limitado de estudiosos que se ocupaban de las investigaciones. Asimismo se potenciaron los departamentos en los que se investigaba sobre cuestiones económicas y se creó un despacho permanente para el censo que tenía que garantizar el momento oportuno en las publicaciones de los datos. Junto al Servicio de Estudios de Bankitalia, hubo otras dos instituciones, el CNR y el ISTAT (Istituto Nazionale di Statistica). Sobre esta última institución volveremos más adelante. 
Antes del estallido de la segunda guerra mundial, el Servicio de Estudios obtuvo un resultado importante con la publicación de dos volúmenes sobre las transformaciones de la economía durante el sexenio 1931-1936. La investigación tenía como objetivo la actualización de los datos contenidos en el Movimento commerciale de 1932 y también la introducción en Italia de elementos de la renta nacional, que iban en contra de la naturaleza de un sistema económico cerrado. Las fases de investigación y la publicación de los volúmenes fueron precedidas y acompañadas por una espesa correspondencia entre el administrador delegado del COMIT, Mattioli, el gobernador del Banco de Italia, Azzolini y el estadístico Giorgio Mortara que fue el director científico. Ugo La Malfa coordinó el grupo de trabajo del Commerciale y Baffi dirigió el grupo de Bankitalia. Los volúmenes fueron publicados en otoño de 1938, sin el nombre de Mortara, que mientras tanto fue obligado a abandonar cada encargo a consecuencia de las leyes antihebraicas. A finales de 1939, el Instituto de Emisión se dotó de un ulterior instrumento para el cálculo de la contabilidad nacional: se trata del Instituto Nacional de Hacienda Corporativa que tenía otra sede diferente de la del Servicio de Estudios. Fue allí donde se examinaron las primeras tentativas de estudiosos privados para dar vida al cálculo de la renta nacional con datos procedentes del Ministerio de Corporaciones, aún no contando con las cifras sobre el valor añadido. ${ }^{3}$ En base a estas valoraciones el ministro de Hacienda, Thaon De Ravel, instaló una comisión de estudio, en la que estaba también Ezio Vanoni, encargada de predisponer una reforma fiscal.

En Italia la valoración de la renta nacional tuvo lugar en la segunda posguerra, y fue hecha posible, por lo que se refiere al componente más importante del valor añadido de la producción industrial, gracias al Censo industrial y comercial de 1937-39, ligado a la figura del estadístico Alessandro Molinari (1898-1962) ${ }^{4}$ Crecido en el mundo intelectual del anarquismo milanés, Molinari elaboró una idea de planificación liberal y socialista. Sus maestros fueron el liberal Luigi Einaudi y el socialista reformista Alessandro Schiavi. Fue director del Istat desde 1929 hasta 1945. En el momento de su nombramiento como director, era presidente del Istat, Corrado Gini, quien intentó en los años veinte definir el criterio de medición de la riqueza de los Estados en el ámbito de la Sociedad de las Naciones. Molinari introdujo en el ISTAT la cultura de los entornos más avanzados del socialismo industrial lombardo y la idea de una programación vinculada al análisis territorial.

\footnotetext{
${ }^{3}$ Véase por ejemplo, A. De Vita, Il reddito nazionale dell'Italia, en «La vita economica italiana», 2 trim. 1939; A. Degli Espinosa, Il reddito e la ricchezza degli italiani nel 1936-37, en «Economia», mayo-agosto 1939; Associazione fra le società per Azioni, Il reddito degli italiani nel 1936, Roma, 1939; P. Thaon di Revel, Indici del reddito nazionale dal 1928 al 1938, en «Atti della Società di Demografia e Statistica», Roma, 1940. Además se publicaron comunicaciones de Lenti, Cosciani e Vinci en el mismo periodo de tiempo.

${ }^{4}$ Sobre la aportación del censo industrial y comercial de 1937-39 a la historia de la renta italiana, puede verse: Misiani, 2007
}

EMPIRIA. Revista de Metodología de Ciencias Sociales. N. ${ }^{\circ}$ 21, enero-junio, 2011, pp. 143-169. ISSN: $1139-5737$ 
De 1936 a 1939, con el inicio de la política autárquica tuvo lugar un extraordinario progreso del sector de las investigaciones económicas, en un cuadro ideológico donde se combinaban elementos culturales muy discordes entre ellos. El proyecto había sido precedido por una fase preparatoria de los criterios de valoración y de actualización de los cuadros técnicos del instituto, ambos llevados a cabo en el cuatrienio anterior. En particular se emprendió un programa para la introducción de máquinas que debían sustentar las operaciones de recuento de los censos; en segundo lugar fue relanzado el programa para la concentración de las funciones de recogida de datos económicos del principal instituto de investigación nacional. Y finalmente se promovió la actualización de la metodología de investigación en la que debía inspirarse la estadística moderna funcional para una política de programación. El censo general de la población de 1936 representó el punto de partida de esta fase, ya que evidenció por primera vez una radiografía de la estructura demográfica capaz de hacer emerger no sólo unos datos generales, sino también la articulación regional y en particular las «zonas estadísticas» que constituían la estructura de Italia. Un aspecto, éste último, que no encontró ningún interés por parte del Régimen fascista.

El censo industrial y comercial de 1937-39 permitió adquirir todos los datos necesarios para medir el sistema de las empresas diversificadas en base al tamaño y la ubicación geográfica en el territorio nacional. ${ }^{5}$ Fue dirigido por Molinari que se valió de la colaboración de Pietro Battara y Eugenio Rienzi. El censo se enfrentó con el problema del cálculo del índice de productividad y el valor añadido; de hecho quería mostrar una visión macroeconómica sin renunciar al objetivo de representar la articulación territorial. Se pusieron los fundamentos de la construcción de la renta nacional siguiendo una corriente que tenía en cuenta la experiencia de la crítica liberal (Battara, 1963).

Los antecedentes en los que se inspiró el censo de 1937 fueron los censos industriales realizados por Alemania y Estados Unidos. El cálculo del valor monetario comportó algunas dificultades técnicas y organizativas, a las que se puso remedio con precauciones prácticas que sólo habrían tenido que ser adoptadas de manera transitoria y no de manera habitual. El primer problema concernía a la dificultad para individualizar los diferentes sectores productivos en el momento de su máxima actividad. Además algunas dificultades organizativas indujeron a fraccionar la encuesta en tiempos diferentes, pero con el deseo de que en el futuro se desarrollasen los censos en la misma fecha, utilizando técnicas que permitieran también censar sectores productivos estacionales. Otra medida práctica, ideada para el censo de tipo industrial, fue la de distinguir en la encuesta de productividad aquellas empresas, - tendencialmente las empresas

${ }^{5}$ La publicación de los resultados del censo, por las circunstancias excepcionales atravesardas en el período bélico, fue interrumpida después del estallido de la guerra y fue reanudada en 194748 y completada en 1950. (Istat, 1937-1950). Los acontencimientos históricos más recientes han confirmado el peso creciente del desarrollo industrial en la determinación de la renta nacional emergida en el censo de 1937: Rey, 1991

EMPIRIA. Revista de Metodología de Ciencias Sociales. N. 21, enero-junio, 2011, pp. 143-169. ISSN: 1139-5737 
grandes - que no tenían interés en dar indicaciones fiscales falsas, de aquellas de carácter artesanal que, en cambio, podían tener interés en dar respuestas no verdaderas. Para estas categorías se preparó un sistema de cálculo deductivo.

En esta decisión emergen dos elementos de carácter general que merecen ser mencionados. El primero era la confianza en la gran industria, un dato que aunaba toda una generación y que ha sido bien resumido en el apoyo al modelo fordista. El segundo factor más relevante era el ser conscientes de que hacía falta asumir un enfoque crítico con respecto a los enfoques oficiales y de la política de Confindustria. Así el censo hizo emerger la tendencia de la industria italiana a la concentración sectorial y territorial, premisa a la que tenía que seguir una legislación contra los monopolios, hipótesis completamente inverosímil. Esta afirmación encuentra confirmación en el diálogo que acompañó las fases de la investigación entre Molinari, el círculo milanés de Mattioli y el grupo clandestino del Partido socialista de Basso y Morandi, continuado desde fuera de Italia con Nenni en París y Sraffa en Gran Bretaña. Piero Sraffa había colaborado con él en la Oficina de Estudios del la ciudad de Milan antes de trasladarse al Reino Unido (Naldi, 2000). En la segunda postguerra, Molinari había importado a Italia, de mundo anglosajón, los conceptos de «Areas en desarrollo» y «Areas con con problemas de empleo» (Unemployment Areas) (Meynell, 1960), en el marco del dibate sobre el desarrollo industrial del Mezzogiorno (Sur de Italia).

El censo tuvo que proveer informaciones atendibles relativas a tres índices significativos del ciclo productivo: la unidad técnica (ejercicio); la unidad local (establecimiento); la unidad jurídico-económica (empresa). El dato más relevante para el cálculo del curso productivo fue el ejercicio, constituido por las elaboraciones industriales que pertenecían a una, y solamente una, de las 283 subclases en las que fueron clasificadas las actividades industriales. Las unidades técnicas fueron indispensables para el cálculo correcto del valor añadido y para garantizar una repartición racional de las actividades industriales en base a la tecnología empleada en el ciclo productivo. Eso permitía analizar las características propias de los muchas ramas empresariales y sus modificaciones en el tiempo. Por cada una de las ramas o subclases en las que fue clasificada preventivamente la actividad industrial, fue dispuesto un modelo de encuesta llamado «formulario de ejercicio» que contenía: una parte de preguntas comunes a todas las empresas y a una parte variable, relativa a las materias primas y secundarias, los ingredientes, los productos y los subproductos, las instalaciones y las maquinarias características. Podía ocurrir, por ejemplo, que en un mismo establecimiento estuvieran presentes dos o más ejercicios, (ejemplo, molinos y fábrica de pastas alimenticias). Para el sondeo de las actividades artesanales se utilizaban formularios más sintéticos. ${ }^{6}$

${ }^{6}$ Por cada una de las 283 subclases fue utilizado un formulario especial y a veces dos: uno para los ejercicios industriales y uno para los artesanos. En el caso de dos o más ejercicios en un mismo establecimiento fue empleado un tercer formulario (formulario de establecimiento) para notar los servicios generales, comúnes a los dos o más ejercicios.

EMPIRIA. Revista de Metodología de Ciencias Sociales. N. ${ }^{\circ}$ 21, enero-junio, 2011, pp. 143-169. ISSN: $1139-5737$ 
En primer lugar, el censo industrial verificaba, como ya se hacía antes pero con mayores detalles, los datos estructurales relativos a la fecha del censo: informaciones generales sobre el ejercicio, personal, fuerza motriz, medios de transporte, instalaciones y maquinarias, etc. ${ }^{7}$ En segundo lugar, producía datos cuantitativos sobre el ciclo de elaboración a lo largo de un año entero denominado año de censo. Este es un momento importante que señala el paso hacia la investigación para determinar el cálculo de la productividad y presenta una indudable novedad en la historia italiana de la estadística aplicada: a) el valor de las materias primas particulares, materias secundarias, ingredientes, etc. aplicados en el año del censo y además todos los productos terminados y subproductos hechos u conseguidos en el susodicho año; b) la oscilación mensual de la mano de obra y las horas de trabajo y sueldos pagados a los obreros; c) consumos de combustibles, energía eléctrica, etc. d) improductividades, a principios y finales del año de censo, de las materias primeras y los productos y subproductos. ${ }^{8}$

En contraste con los edictos de la retórica del Régimen, la realidad que resultó de las investigaciones del ISTAT indicaba una Italia invertida por el proceso de modernización, aunque quedaban - y más bien se acentuaban - las diferencias entre niveles productivos de la industria y la agricultura, que indicaban la diferencia regional entre Norte y Sur. De la investigación aparecieron las primeras señales de reactivación conducidas por el sector industrial y comercial, acompañadas por una marcada tendencia a la concentración. Al mismo tiempo, los datos del censo mostraban las señales de una diferencia territorial en el desarrollo entre las regiones septentrionales y el Mezzogiorno de Italia, con efectos inmediatos en la ocupación (Tattara y Toniolo, 1975).

Así, mientras el Régimen proclamaba en sus escritos la conclusión de la cuestión meridional, los datos mostraban una Italia muy diferente. Existía un desnivel de productividad en las regiones meridionales al que correspondía un paro estructural no reabsorbible con una política de industrialización (Lenti L.,1943). A causa de la crisis bélica el recuento del censo industrial fue suspendido y el censo general de la agricultura planeado para el año 1942, no tuvo lugar. Las líneas de investigaciones que se habían programado en la época fueron reanudadas y actualizadas una vez que se abandonó el sistema cerrado del corporativismo, y se pusieron en práctica a partir de la elaboración de los primeros planes para la

${ }^{7}$ Por lo que concierne los datos estructurales, Molinari dio particular importancia a las voces encargados, (empresarios y eventuales coadyuvantes, ejecutivos, empleados, obreros y figuras parecidas), y fuerzas motriz, (f.m.). Esta voz fue expresada en caballo de vapor (CV) utilizable para el funcionamiento de la maquinaria de todos los ejercicios «censados», activos o no a la fecha del censo. La fuerza motriz fue determinada de modo que se evitaran duplicaciones de potencia: para la medición de la potencia de los motores primarios fue sustraída la potencia de los generadores de energía eléctricos y otras fuentes de energía. Molinari, 1942 y 1947.

8 El censo tuvo lugar en 1937, (pesca y industrias alimenticias); en 1938, de las demás industrias a excepción de las mecánicas, y en 1939 de la industria mecánica. Las industrias estacionales fueron censadas durante los meses de normal actividad. En 1938 también fueron censados «transportes y comunicaciones» y en 1939 (31 de octubre), el «comercio».

EMPIRIA. Revista de Metodología de Ciencias Sociales. N. ${ }^{\circ}$ 21, enero-junio, 2011, pp. 143-169. ISSN: 1139-5737 
reconstrucción, y a partir de 1948, en el ámbito de las discusiones surgidas alrededor de la aplicación del Plan Marshall.

\section{EL ORIGEN DEL REFORMISMO LIBERAL}

A partir de 1942-43 se reconoció, como también ocurrió en las democracias liberales, la necesidad de dotar al país de una contabilidad nacional para combatir la inflación y defender el poder adquisitivo de los ciudadanos (Caracciolo, 1993). La causa de la inflación se debía a la prolongación de la guerra y de hecho se reconoció la exigencia de emprender una política de desarrollo para evitar el colapso del sistema productivo que habría tenido consecuencias sociales dramáticas. De acuerdo al proyecto pangermano que anhelaba una planificación dentro de un mercado europeo cerrado, Italia habría tenido que dirigirse hacia un sistema de intercambios internacionales abiertos. Estos temas fueron destapados sólo tras el final de la guerra. Después de la liberación de Roma se puso en marcha sin éxito el programa, ya adoptado por Gran Bretaña y Estados Unidos, que promovía la investigación sobre la contabilidad nacional para disponer de un sistema de defensa contra la inflación generada por la guerra. En 1944-47 Italia fue excluida del programa emprendido por las Naciones Unidas para normalizar el cálculo de la renta nacional, en función de la aplicación de los acuerdos de Bretton Woods. Las razones hay que buscarlas en la dificultad para la transición a la democracia. Después de la liberación de Roma, el ISTAT había colaborado con el Comando Militar aliado a un primer cálculo de la contabilidad económica y social en las regiones liberadas para defenderlas de la inflación, pero se trató de una tentativa que no tuvo una aplicación concreta. Más bien, el estudio sirvió para elaborar un documento de la UNRRA (Administración de las Naciones Unidas para la Reconstrucción) sobre las necesidades de Italia y fue utilizado en la Conferencia de paz de agosto 1946 como base para negociar la entrada de Italia en el sistema de Bretton Woods. ${ }^{9}$

En estos meses se abrió en el ISTAT una fase de grave trastorno interior que perjudicó su capacidad de funcionamiento. El ataque radicaba en la lucha ideológica y también en el emerger del espíritu conservador de la burocracia. Esta disputa llevó a la destitución del director del ISTAT, Molinari, y al abandono del grupo de trabajo de cultura liberal y laborista. Molinari encontró colocación en la SVIMEZ (Associazione per lo sviluppo dell'industria nel Mezzogiorno), in-

${ }^{9}$ La Misión italiana del UNRRA engendró una Division of Operational Analysis-Economic Analysis Branch. Colaboraron en ella, además de Molinari, también Guglielmo Tagliacarne como representante de la presidencia del consejo, ya de la Confederación de los Comerciantes, y Guglielmo Mancini, del Banco de Italia, Enrico Boccia, del Istat y Giuseppe Montanaro, de la Cámara de Comercio italiana para América. Las conclusiones de los estudios proveyeron la base para un estudio acerca de la situación económica de Italia en 1947, dibatida en la V sesión del Unrra que tuvo lugar en Ginebra entre el 5 y el 6 de agosto de 1946. Unrra, Survey of Italy's economy, 1947.

EMPIRIA. Revista de Metodología de Ciencias Sociales. N. ${ }^{\circ}$ 21, enero-junio, 2011, pp. 143-169. ISSN: $1139-5737$ 
stitución nacida en diciembre de 1946 que desempeñó un papel de primera importancia en la discusión acerca de la programación. En verano de 1945, Benedetto Barberi asumió la regencia del ISTAT. Había entrado en el ISTAT a mediados de los años treinta, gracias al auxilio del Partido Fascista y enseguida fue nombrado jefe del departamento de estudios. Fue un clásico tecnócrata, dotado de ambición personales e indudables capacidades técnico-científicas, fue regente y luego director general hasta 1963. El presidente del ISTAT en la posguerra fue el católico Cataletti Gaudenti, una figura de segundo plano resultado del acuerdo entre demócratas cristianos y comunistas. ${ }^{10}$

El problema del cálculo de la renta nacional fue afrontado por la Comisión Económica promovida por el socialista Pietro Nenni, ministro de la Constituyente. La línea del censo industrial de 1937-39 se difundió en los trabajos de la Subcomisión de la Industria de la Comisión Económica presidida por el rector de la Bocconi, el economista Giovanni Demaria y animada por las generaciones más jóvenes que participaron en los proyectos de reformas de los años treinta. En la relación de la Comisión Constituyente se consideró la idea de adoptar en Italia el «libro blanco», cuya utilidad era la de hacer previsiones para el futuro inmediato y proveer al poder público la ocasión para trazar las líneas de la política económica. La estadística cuantitativa tenía que ofrecer a la política las herramientas para una información que fuera lo más objetiva posible, en antítesis a la propaganda de los totalitarismos. Se crean los fundamentos de un lenguaje político común tanto al liberalismo de Einaudi como al socialismo laborista. Esta concepción de la función de la estadística pública fue completamente incompatible con una planificación de tipo soviético (Bettelheim 1949), asimismo había sido perjudicada por el régimen antiliberal del Fascismo.

Sólo después de la apertura de la democracia hacia el comunismo, la situación empezó a aclararse. Durante el verano 1947, con el Plan Marshall se inicio en el mundo occidental la edad de oro de la estadística econométrica y se extendió con la difusión de la cultura de la programación. Se adoptó el principio de fundar la política económica en base al cálculo de la renta y la contabilidad nacional. Las Naciones Unidas emprendieron una comparación internacional, luego reanudada en europa, para unificar la terminología relativa a los balances económicos nacionales e iniciaron una comparación entre los expertos de los países del área occidental con la implicación de la Asociación Internacional de estadística. ${ }^{11}$ Esta línea de cambio también fue acogida en Italia tras la constitu-

${ }^{10}$ Molinari propuso la candidatura de Alessandro Schiavi, que antes no había sido considerada. En una carta del 16 abril 1945, Einaudi le preguntó al jefe del gobierno Bononi que no firmara el nombramiento. El cargo de presidente era el papel de mayor prestigio por un estadístico. Correspondió a estudiosos de fama internacional y dotados de capacidades organizativas. Indicó como alternativa los nombres Costanti Bresciani-Turroni, Pasquale Jannaccone, Giorgio Mortara o Umberto Ricci. L. Einaudi a I. Bonomi, 16 aprile 1945. ASBI, Presidenza della Repubblica Italiana.

${ }^{11}$ El fruto de esta comparación internacional fue la publicación por parte de la ONU del volumen, National Income Statistics 1938-1948, 1950.

EMPIRIA. Revista de Metodología de Ciencias Sociales. N. ${ }^{\circ}$ 21, enero-junio, 2011, pp. 143-169. ISSN: 1139-5737 
ción del IV gobierno De Gasperi, surgido a finales de mayo de 1947, e interpretada por el ministro de Finanzas y Vicepresidente del Consejo de Ministros, Luigi Einaudi. Se concertó la operación con el director general de Bankitalia Donato Menichella, luego gobernador del mismo instituto desde enero de 1948 hasta agosto de 1960. Esta decisión puso en evidencia la dificultad de seguir el camino indicado por la Comisión Económica, para insertar el tema del balance económico nacional en la Carta Constitucional como punto central de una reforma de las instituciones. En tales circunstancias, el Partido Socialista, al contrario de los demás partidos de la izquierda occidental, no siguió el camino de una evolución en sentido laborista y concluyó una alianza con el partido Comunista de Togliatti. Los estadísticos y economistas de cultura laborista colaboraron con el Ejecutivo sin alinearse con una u otra coalición política: operaron en la SVIMEZ y dieron voz a su posición en revistas de frontera como Critica sociale, Mondo Economico o también en el semanal Il Mondo.

En estos meses Einaudi promovió una acción legislativa para llegar a un informe anual sobre la renta nacional. Se trata de la reforma de las reformas que habría tenido que dar sustancia y contenido al Ministerio del Balance ${ }^{12}$. En el proyecto de Einaudi, enunciado el 18 de junio de 1947 a la Asamblea Constituyente, se atribuía a los departamentos técnicos del ministerio del Balance la competencia para redactar la informe general anual. Einaudi hizo referencia al «libro blanco» inglés, que había sido dividido por él mismo en dos partes: una «conoscitiva», redactada por estadísticos que habían sido interpelados por el ISTAT; y otra «practicable», capaz de proveer los datos sobre la política de desarrollo (L. Einaudi,1947). En realidad el ministerio del Balance no tuvo un valor efectivo y tras el nombramiento de Einaudi a Jefe del Estado sólo fue un nombre sin algún contenido, dependiente del Tesoro. En estos meses el socialista reformista Tremelloni, responsable del CIR, se ocupó del tema de la contabilidad nacional y lo conectó al programa de política de pleno empleo.

En otoño de 1947 el CEN (Consejo Económico Nacional), organismo técnico-científico dependiente del CIR, creó la Comisión para el estudio de la renta nacional con el objetivo de redactar el balance económico italiano. La comisión estaba constituida por estudiosos y técnicos y seguía la organización de las Subcomisiones del Ministerio de la Constituyente. El CEN encargó al ISTAT la recogida y elaboración de los datos de la renta nacional y lo sometió a una vigilancia externa. Dentro del Instituto de Estadística Pública se instituyó una Comisión econométrica y empezó un proceso de reorganización interior en función de las nuevas tareas (Guarini,2003). Fue en este contexto cuando Antonino Giannone entró en el Instituto, y en el mismo período Pietro Battara también fue llamado de nuevo a trabajar allí. En verano de 1948 el ISTAT presentó un primer esquema redactado bajo la dirección de Barberi, aunque sin duda, los datos to-

${ }_{12}$ Ministero del Bilancio. Ministerio responsable de la Contabilidad Nacional, podría traducirse como Ministerio de Finanzas, aunque no exactamente. (NT)

EMPIRIA. Revista de Metodología de Ciencias Sociales. N. ${ }^{\circ}$ 21, enero-junio, 2011, pp. 143-169. ISSN: $1139-5737$ 
davía presentaban numerosas lagunas; bastante deficiente era la parte relativa a la Administración Pública. ${ }^{13}$ En estos meses, el Comité Italiano para el Estudio de la Renta y la Riqueza, presidido por Gini, ${ }^{14}$ empezó a desarrollar su trabajo incorporando una cierta profundidad teórica en relación a los organismos internacionales. En esta delicada coyuntura se programó la primera normalización del ISTAT con las instituciones de la Italia republicana.

De Gasperi había encargado a Einaudi para que examinara, junto con el Vicepresidente del Consejo Saragat y el ministro Fanfani, el esquema de la disposición legal. En calidad de responsable de Finanzas, Einaudi no quiso encontrarse con el director del ISTAT Barberi y nunca visitó el Instituto. ${ }^{15}$ En estas condiciones la medida para volver a una situación de orden, promovida por los servicios estadísticos, fue aprobada sólo porque se restablecieron las condiciones anteriores a la guerra. El nuevo ordenamiento del ISTAT fue el fruto de una concertación directa entre Barberi y la Contabilidad general del Estado, mientras que Einaudi no tomó parte en ello. Entró en funcionamiento en 1949 sin las reformas necesarias a las nuevas tareas y sin intervenir en el problema de la dependencia de la Presidencia del Consejo planteado por Mussolini. El único elemento de novedad fue el restablecimiento del Consejo Superior, organismo con funciones de investigación científica que había sido suprimido por el Fascismo durante la guerra. La elección de Lanfranco Maroi como nuevo presidente sancionaba la voluntad de volver a aproximarse a la estadística municipal prefascista. Maroi fue presidente del ISTAT de 1949 a1961, y en el Consejo Superior de Estadística también entraron especialistas próximos al entorno milanés como Pier Paolo Luzzatto Fegiz, Guglielmo Tagliacarne, Mario Saibante, Ferdinando De Fenizio y Libero Lenti que habían fundado el Centro de Estudios para la Coyuntura en el ámbito del Instituto de Estudios Económicos (ISE). ${ }^{16}$ Esta fase de transición se acabó en 1953 con la producción de la renta nacional.

${ }^{13}$ Véase Il reddito nazionale e la bilancia dei pagamenti internazionali dell'Italia nel 1938 e nel 1947. Los datos fueron adelantados por Barberi en un artículo publicado en la revista «L' industria» de De Fenizio al final de 1947: Barberi, 1947 y también 1948 y1949.

${ }^{14}$ En la primera reunión del Comité hubo la elección de los cargos sociales: en la presidencia fue elegido Corrado Gini, junto a dos vicepresidentes, Papi y Lenti, y un secretario, Saibante. El comité se reunió alrededor de la Facultad de Ciencias Estadísticas, en calle de las Termas de Diocleciano. Los primeros argumentos afrontados fueron los siguientes: un esquema de las categorías con las que clasificar la renta (relator Saibante); la encuesta de los términos recalcitrantes, (relatores Occhiuto y Giannone) y el examen de la valoración de la renta de 1947 hecho por el Istat.

${ }_{15}$ Véase: las cartas de B. Barberi a L. Einaudi, en las fechas de 8 diciembre 1947, 18 marzo 1948 y 10 agosto 1949. Fundación Luigi Einaudi, Archivio Luigi Einaudi, Sección Corrispondencia, fasc. «Barberi Benedetto»

${ }^{16}$ El ISE se constituyó en Milán en el verano del 1946 por antiguos accionistas y exponentes del socialismo antitotalitario. El presidente fue Ferruccio Parri, pero la dirección efectiva fue dada a Lenti y De Fenizio. El ISE publicaba un boletín, dirigido por Lenti, sobre la coyuntura económica y también textos a favor de las ayudas Erp.

EMPIRIA. Revista de Metodología de Ciencias Sociales. N. ${ }^{2}$ 21, enero-junio, 2011, pp. 143-169. ISSN: 1139-5737 


\section{3. «HIC SUNT» LEONES}

El primer balance económico italiano, caso único en el mundo, no fue discutido en el Parlamento italiano sino en el Senado de Estados Unidos. Se trata del Country Study, producido en febrero de 1949 por el ECA de Hofmann para evaluar el impacto de los fondos Erp en muchos países europeos. El documento presentaba imprecisiones evidentes, que reflejaban la opinión de los economistas alineados en posiciones de ortodoxia keynesiana y dotados de un escaso conocimiento de la estructura económica italiana. En particular el texto, que había sido redactado en base a datos procedentes de fuentes italianas y ensamblados de manera sumaria, acusaba a la política de estabilización monetaria de excesiva prudencia. A las deficiencias institucionales puso remedio, aunque en vía transitoria, el informe del Gobernador del Banco de Italia presentado el 31 de mayo de 1949 (Cotula E. Y otros 1997,55-71). El documento utilizaba los datos del ISTAT de 1938 y era el resultado del trabajo del Departamento de Estudios de Baffi y de la aportación de los expertos italianos y economistas internacionales. En base a estas valoraciones, el Instituto de emisión produjo un programa de inversiones que contribuyó a orientar la política del gobierno, y una vez alcanzada la estabilización monetaria, Bankitalia propuso un plan de inversiones que, en otoño 1949, recibió la aprobación de los institutos de Bretton Woods y se convirtió en un programa de política económica (De Cecco Bini 2006 y Barucci 2008).

Los datos plantearon una acalorada discusión sobre la manera de aplicación en Italia del «multiplicador de las inversiones» de matriz keynesiana. Con razón, Bresciani Turroni evidenció los elementos de diversidad de la estructura del mercado laboral en Italia en comparación con la situación de Estados Unidos, para concluir, aun equivocándose, que el concepto keynesiano del multiplicador en Italia no podía ser aplicado con éxito. El planteamiento del informe de Menichella fue acogida por el VI gobierno de De Gasperi, constituido en febrero de 1950, y fue en estos meses cuando finalmente se llegó a una resolución del problema relativo a la contabilidad nacional.

Durante el bienio 1950-51 el balance económico italiano entró en el ordenamiento ordinario adoptando una solución en contraste con el diseño einaudiano. El proyecto de Einaudi fue acogido en parte por la ley «Ruini-Paratore» del 21 agosto 1949 que le confiaba al ministro del Tesoro la presentación de una relación general sobre la situación económica del país, sin embargo, el problema del reparto de competencias entre las instituciones encargadas de redactar el balance quedó sin solucionar. A principios de 1950, el ministro del Tesoro Pella presentó en el Parlamento la primera relación general relativa a 1949, preparada por los expertos del CIR valiéndose de los datos provistos por el Banco de Italia y la Contabilidad del Estado. El documento mostró evidentes lagunas respecto de los balances de los principales estados europeos y los Estados Unidos. Mientras tanto, en el verano de 1950 el cálculo de la renta nacional del ISTAT alcanzó una primera sistematización. El planteamiento del ISTAT se distinguió de la línea

EMPIRIA. Revista de Metodología de Ciencias Sociales. N. ${ }^{2}$ 21, enero-junio, 2011, pp. 143-169. ISSN: $1139-5737$ 
adoptada por el informe del Banco de Italia ya que consideraba como indicador los productos en lugar de las inversiones, retomaba de manera tradicional las reglas fundamentales en la compilación de una contabilidad nacional contenidas en un manual OECE, ya enunciadas por Stone. ${ }^{17}$ El producto neto se calculó según el «coste de los factores» y el «precio de mercado» con toda una serie de incógnitas que sólo en algun otro lugar pudieron ser solucionadas. ${ }^{18}$ El problema principal relativo a la aplicación del modelo de Cambridge concernía al cálculo del producto interior de la administración pública, ${ }^{19}$ pero sobre todo era inadecuado para representar la estructura y problemas de Italia.

A pesar de estos límites evidentes De Fenizio propuso una modificación de la ley Ruini-Paratore a favor del ISTAT (De Fenizio 1950). El Ministro del Te-

17 En septiembre de 1944, al acercarse el fin de la guerra, se desarrolló a Washington un mitin del Sub-committee of National Income Statistics of the League of Nations compuesto por los expertos de Estados Unidos, Gran Bretaña y Canadá, sobre el tema de la estandarización de la renta como soporte del nuevo orden económico mundial. El grupo le confió a Stone la redacción de un memorándum, que fue aprobado por el Sub-committee reunido en Princeton en diciembre de 1945, que mientras tanto se había extendido hasta incluir Australia, Holanda y México. El grupo asumió la denominación de Statistical Commission adherente al Economic and Social Council de las Naciones Unidas y presentó el resultado de su trabajo en la relación publicada en febrero de 1947. La implicación de Italia empezó en el período siguiente, con el inicio de una fase de estandarización a obra de la OECE, con sede en París, en el cuadro de la aplicación del Plan Marshall. De 1949 a 1951 Stone reunió a Cambridge un grupo de investigación sobre la renta nacional que confeccionó un modelo de contabilidad simplificada adoptado por Italia y Francia (OECE 1952 y Vanoli 25-45 y 130-135). Este modelo fue considerado como la Biblia por el grupo de Cambridge, tal como el economista francés Francois Perroux escribió y fue utilizado como medida de comparación para juzgar los resultados alcanzados en muchos países. En particular la renta italiana fue valorada por el economista Jean Marczewski que dio su aprobación, pero criticó los elementos diversidad con respecto del national income de Cambridge: 1) la inserción de la voz acerca de las remesas de los emigrantes y las donaciones; 2) la mayor diferencia afectaba al tratamiento del gasto de la Administración Pública. Ese elemento reflejaba un planteamiento diferente relativo a la relación entre las familias, los consumidores y el Estado. El modelo estandardizado ofrecía la posibilidad de alcanzar el objetivo de un estandarización de los países, mientras que no examinó el problema de los criterios y los límites de aplicabilidad de la nueva metodología para representar la riqueza del País (Vanoli 2005 45-65).

${ }_{18}$ El valor añadido era obtenido por la diferencia entre el valor de los precios de mercado y el valor de las materias primarias y secundarias y otros materiales y servicios empleados por la producción. El producto neto «al coste de los factores» también se refería a precios de mercado, y representaba la suma de lo que se debía a los factores de la producción, a lo bruto de los impuestos directos.

${ }^{19}$ Dentro de la voz de la Administración Pública se incluyeron las administraciones centrales y periféricas y los entes no profit ?, mientras que quedaban excluidas las empresas del Estado de carácter industrial, las empresas del grupo IRI, pero también los Ferrocarriles del Estado. En la voz Administración Pública la renta se sacó de la suma de los sueldos así como de las rentas de los edificios y otros inmuebles sin fines de lucro. Del cómputo quedaba excluida la renta procedente de los edificios de la Administración Pública, así como no se calcularon los intereses sobre la deuda pública. Barberi declaró que a causa de la falta de colaboración por parte de los funcionarios de la Contabilidad, y también a causa de los criterios disconformes empleados para la compilación de los bilance, esta operación fue muy ardua.

EMPIRIA. Revista de Metodología de Ciencias Sociales. N. ${ }^{\circ}$ 21, enero-junio, 2011, pp. 143-169. ISSN: 1139-5737 
soro tuvo que aplazar la fecha de presentación por algunos meses (abril en lugar de enero) y sobre todo, tuvo que esperar a que el ISTAT redactara el balance consultivo y que el CIR presentara la estimación para el año siguiente valiéndose de las cifras del ISTAT. En este esquema en el que estaban representadas las instituciones económicas así como el Departamento de Estudios del Banco de Italia y el Tesoro, el ISTAT adquiría un peso más relevante respecto al CIR. Einaudi se procuró el texto de la memoria y decidió escribir un duro comentario. ${ }^{20} \mathrm{El}$ texto del Jefe del Estado es de indudable interés y merece que recojamos algunas de sus partes esenciales pues Einaudi no confiaba en el ISTAT e instó a seguir el ejemplo de Holanda que, aunque no disponía de un instituto competente, había creado un departamento ad hoc dirigido por Jan Tinbergen. ${ }^{21} \mathrm{El}$ ministro del Tesoro tuvo que elegir un Tinbergen italiano y «construir un falso ente destinado a morir el día que desapareciera la posibilidad de valerse de aquella persona» (Di Fenizio,1950,4) Pero sería mejor sería si no fuera un funcionario cualquiera pues en ese caso, «tendremos una de las usuales cosas insípidas que no aclarará el problema». Más adelante continúa: para solucionar el problema de la competencia se tiene que animar a muchos hombres y despachos públicos y privados para que recopilen, en competición entre ellos, cada uno su propio balance. «Quien tenga más hilo (de lógica y de modestia), hará más tela; y también puede ser que un balance realizado por un particular obtenga mayor consenso que otro balance, provisto del carisma de la oficialidad» (Di Fenizio 1950,17). Esta posición expresaba muy bien su sentimiento antiburocratico y en particular la intolerancia hacia alguna «presunción profesional» favorable a una interpretación mecánica de un pensamiento keynesiano funcional a los intereses del poder.

Las discusiones sobre el ente al que encargar el informe resultaron secundarias, lo que realmente preocupaba era el contenido sustancial de la renta nacional. Presentó una serie de críticas teóricas recogiendo las investigaciones de economistas liberales como Hayek y John Hicks. El jefe del Estado le aconsejó al alumno que desplazara la mirada del modelo abstracto a la realidad en sus aspectos concretos. La renta nacional para ser creíble tenía que reconocer de manera explícita los límites de la contabilidad de algunos aspectos de la realidad. Además, no tenía que perder de vista la dimensión que podríamos definir microeconómica respecto a la mirada macroeconómica. La estadística podría con-

${ }^{20}$ A proposito di una memoria del prof. di Fenizio, un escrito de L. Einaudi enviado a F. Di Fenizio, 25 de julio 1950. Fondazione Luigi Einaudi, Archivio Luigi Einaudi, F. «Di Fenizio Ferdinando». Fondazione Luigi Enaudi, Archivio Luigi Einaudi, Corrispondenti, f. «Di Fenizio Ferdinando».

${ }^{21}$ En un primer momento, la compilación del balance económico fue confiada al Netherlands Central Bureau of Statistics (el Instituto Nacional de Estadística), para luego crear, en la posguerra, un despacho a hoc: el Netherlands Central Planning Bureau. Gracias a Tinbergen y su despacho, Holanda consiguió relevantes resultados y alcanzó el objetivo: ser en 1949 el único País que agrega en el balance económico el balance del Estado obtenido con los resultados de los diferentes despachos de la Contabilidad.

EMPIRIA. Revista de Metodología de Ciencias Sociales. N. ${ }^{\circ}$ 21, enero-junio, 2011, pp. 143-169. ISSN: $1139-5737$ 
siderarse un instrumento fundamental para la decisión pública, a condición de que fuera capaz de coger los cambios de la realidad económica y social, poniéndo ante los entrevistados las preguntas adecuadas. De hecho, el acto cognoscitivo tenía que ser capaz de ofrecer respuestas satisfactorias a las preguntas del «hombre común», con el que el jefe del Estado se identificaba (Di Fenizio,1950,4).

Antes que omitir o improvisar valores, vitales en la dinámica de la economía hacía falta insertar la categoría y poner la mención hic sunt leones, tal como lo ponían en los mapas los geógrafos de la antigüedad para indicar los lugares de los que no se tenían noticias. La recepción del lema hic sunt leones, «serviría para decirles a aquellos hombres, que constituyen la mayoría de los lectores o presuntos lectores de estos documentos, que hay en este mundo muchas cosas que se ignoran porque no se pueden conocer y que sería un acto de soberbia pretender conocer». ${ }^{22}$ Desde luego esta es la parte más actual de su razonamiento. Hoy, a la luz de la crisis de 2007, estas mismas observaciones podrían aplicarse por ejemplo al caso geográfico de China, India y los países en desarrollo de la zona latinoamericana, sobre la que sabemos muy poco. Este discurso también puede entenderse en sentido figurado para representar las variables de las que se desconocen las medidas y que inciden en la economía del presente y del futuro. La renta inglesa, a la que se refería el documento del ISTAT, presentaba conceptos ambiguos como la «producción bruta al coste de los factores». ${ }^{23}$ Einaudi estaba perplejo acerca de la posibilidad de calcular las inversiones a partir de la sustracción de los consumos, además había categorías no controlables. ${ }^{24}$ Temía

${ }^{22}$ Id., p. 7. En otra parte se lee: «Aquí en Italia una condición esencial para que el primer balance que será publicado sea una cosa seria es que ello esté lleno, en la mayor parte de sus categorías, de las palabras «hic sunt leones» [...]», A proposito di, cit. p. 13. La sugerencia fue propuesta, algunos días antes, a margen del comentario de la relación de Bankitalia de 1949. Eso confirma el carácter no provocador de su propuesta. L. Einaudi a D. Menichella, 3 julio 1950. ASBI

${ }^{23}$ La definición fue tomada por Barberi del balance inglés. Se refiría a aquella cierta cifra a la que hacía falta añadir la suma de los impuestos indirectos y de la que sustraer las subvenciones para precios políticos y llegar a conseguir la cifra de la renta nacional al precio de mercado. En Italia, pero no sólo, hubo una objetiva dificultad de cuantificar las entradas de los impuestos indirectos. Einaudi llegó a la siguiente conclusión: «¿Cuál es la razón de todo este engaño contable si no la de satisfacer a la presunción de algún profesor encerrado en un despacho estadístico?». A proposito della memoria, cit. p. 5.

${ }^{24}$ Notó una ulterior incongruencia relativa a la elección de alejar del balance a las tareas domésticas, los servicios desempeñados para si mismos por las personas de familia. Un problema aún hoy en día no solucionado. Como también el de la suma bruta de casas alquiladas. Incluso notó que en Italia, a diferencia de Inglaterra, existía una ley de vínculo a los alquileres que inducía a subestimar la categoría. La suma debía ser decuplicada respecto a la percibida por los propietarios. Sacó ulteriores ejemplos del examen del formulario predispuesto por el ISTAT para el censo general de la agricultura que habría tenido que tenerse en 1950, en concomitancia con el censo mundial promovido por la FAO. Les pidió a los campesinos el declarar al departamento agrícola competente la suma de los productos agrícolas y de los animales, no teniendo en cuenta su propensión a la evasión fiscal. Este ejemplo redujo la confianza en las cifras de la renta nacional. En realidad, como el mismo Barberi escribió, las categorías del formulario fueron copiadas de las del censo propuesto por la FAO. Por las dudas levantadas y la dificultad de solucionarlas en breve, el censo de la agricultura fue pospuesto.

EMPIRIA. Revista de Metodología de Ciencias Sociales. N. . 21, enero-junio, 2011, pp. 143-169. ISSN: 1139-5737 
el surgimiento de una estadística totalitaria basada en la ilusión de llegar a un control total de la realidad. De sus palabras filtraba un cierto escepticismo hacia el plantamiento macroeconómico, pero también de la invitación a introducir correctivos de corte liberal con los que reducir el margen de error. «[...] Hace falta redactar un esquema lógico y completo, escribir todas las palabras del modo más claro posible, evitando los misterios y las complicaciones procedentes de la presunción profesional, conformándose a las exigencias de aquellos hombres comúnes que se llaman políticos, parlamentarios, periodistas etc. etc. y no tener la soberbia de conocer lo que se ignora» (Di Fenizio 1950,26-27). Para favorecer «la manía del hombre común de conocer los totales» también se podía cometer algún «pecado capital» (Di Fenizio 1950,16-17), pero se tenían que escribir los datos inciertos con letras de imprenta muy bien distinguibles a los que tendrían que seguir uno o más signos de interrogación, según una regla ya utilizada en las revistas científicas. Para evitar que «el hombre común»se ofendiera y acusara de ignorancia a los departamentos estadísticos, los redactores habrían tenido que añadir aún con mayor evidencia que «sólo la ignorancia del público pretende poseer datos seguros acerca de aquellos términos sobre los que no existen y no pueden existir noticias». En fin esta precaución habría aumentado el grado de autenticidad de la renta nacional y la confianza de la clase política y los medios de comunicación hacia «aquellos beneméritos investigadores que sólo les comunican las noticias que poseen, y no las que deberían inventarse para satisfacer una curiosidad infantil» (Di Fenizio 1950 16-17). La información estadística tenía que ser fiable y de fácil acceso, principal elemento de garantía de una democracia liberal y de freno contra los totalitarismos. ${ }^{25}$

Para aumentar el margen de autenticidad de la renta nacional hacía falta restablecer un contacto directo con la viva realidad del país, refiriéndose a la tradición de las investigaciones económicas y sociales. Algunas categorías de inversión que constituían la riqueza de Italia se escapaban del cálculo de la contabilidad económica. Viajando en tren o en coche ¿cuántos techos nuevos con tejas flamantes se podían descubrir? ¿Qué parte de todo esto era valorable y qué no? Muchas de estas construcciones habían sido edificadas sin el respeto de las

${ }_{25} \mathrm{Al}$ respecto, de notable interés es la carta escrita por Einaudi, en calidad de jefe del Estado, a Alcide De Gasperi, a pocos días de las elecciones políticas, el 14 de marzo 1953. De Gasperi le había enviado al presidente del ISTAT Maroi una carta en la que se prohibía la publicación de las estadísticas sobre la producción industrial aduciendo razones de seguridad militares, y se anunciaba el envío de oficiales especializados y la institución de una comisión militar con tareas de control sobre el trabajo del ISTAT (n. 46344/10026 del 11 de marzo1953). Einaudi decidió reaccionar: «La discreción que circunda aquellas noticias en los países más allá de la «telón de acero», no creo sea un beneficio para los países interesados. Supuestamente las dos últimas grandes guerras mundiales fueron vencidas por aquellos países en los que las noticias sobre la producción industrial estaban circundadas por el mínimo secreto - añadió - Si eso ocurriera y se conociera - y sería imposible evitar la notoriedad de tal hecho - las estadísticas publicadas por el instituto Central perderían cualquier crédito». (L. Einaudi a A. De Gasperi, 14 marzo 1953. ASBI, Presidenza della Repubblica Italiana. Segreteria privata del presidente, ua. 6). Esta iniciativa no tuvo ninguna continuación.

EMPIRIA. Revista de Metodología de Ciencias Sociales. N. ${ }^{\circ}$ 21, enero-junio, 2011, pp. 143-169. ISSN: $1139-5737$ 
licencias constructoras, estaban sin permisos, como incluso las considerables mejorías agrícolas e intervenciones en el mundo rural. Todo esto era el fruto de ahorros efectivos que casi nunca pasaron por el banco ni siquiera por la forma monetaria; sin embargo, una vez concluidas, se trataba de inversiones que en un balance nacional debían ser enumerados, aunque debían ser añadidas las palabras hic sunt leones. De Fenizio acogió todas estas observaciones del jefe del Estado, y las insertó como un correctivo del modelo de Cambridge de contabilidad nacional (Di Fenizio, 1950). ${ }^{26}$

La definición de la renta nacional dió un paso adelante con el censo general de la población del 4 noviembre 1951, al que también se unió el segundo censo de la industria y el comercio. El ISTAT alcanzó este fundamental objetivo al precio de algunas renuncias. El censo de la industria y el comercio de 1951, a diferencia de el de 1937-1939, calculó el valor añadido tomando en consideración los productos, sin censar el factor tecnológico, ${ }^{27}$ y además sólo limitó la investigación a algunos productos industriales. En estos años la principal aportación al conocimiento de la realidad económica no vino del ISTAT sino de la Encuesta Parlamentaria sobre el Paro, que profundizaba sobre la cuestión de la población entendida como fuerza de trabajo.

En 1950 Tremelloni llevó al Parlamento la cuestión de la necesidad de un censo sobre el paro en función del inicio de una política de plena ocupación, según indicaban las Naciones Unidas. Los socialistas y los comunistas se alinearon en contra, con la parcial excepción de la CGIL. Hasta entonces los datos oficiales se sacaban del Ministerio del Trabajo, que se basaba en los inscritos en las oficina de empleo. ${ }^{28}$ Este criterio tenía naturaleza administrativa y era insuficiente para promover una política de plena ocupación. En 1951 el ISTAT decidió incluir la categoría de «paro» en una encuesta periódica sobre la fuerza de trabajo, pero adoptó el criterio estadounidense con el resultado de subestimar el fenómeno; pues en Estados Unidos el sondeo de datos se desarrollaba en los lugares de trabajo, y este criterio era eficaz para expresar el fenómeno en su periodicidad, pero inadecuado para destacar el paro estructural que afligía Italia.

${ }^{26}$ Las tesis presentes en el escrito fueron adelantadas en una carta reenviada por De Fenizio a Einaudi el 2 agosto de 1950. En estos años, se publicaron algunas de las más importantes contribuciones teóricas de Battara sobre la renta nacional en las que las dudas de Einaudi encontraron sistematización como aportación a la fundación de un reformismo liberale (Battara, 1950 y 1955).

${ }^{27}$ Limitó la encuesta a las unidades locales (el establecimiento), y jurídicas (la empresa), mientras que no proveyó noticias sobre la unidad técnica (ejercicio), o bien los datos relativos a las elaboraciones industriales de una de las 283 subclases en las que el ISTAT había clasificado las actividades industriales

${ }_{28}$ El Ministro del Trabajo Fanfani restableció la medición periódica del trabajo, retomando una iniciativa que se remontaba al nacimiento del ministerio en 1920. En 1947 instituyó una Comisión de las estadísticas del trabajo que trabajó hasta 1959. Varias consideraciones también se hicieron acerca de los miembros de las listas de colocación con la inclusión de los trabajadores estacionales, mientras que el censo americano se propuso aislar el fenómeno del subempleo relacionado con el curso del mercado del trabajo. Además el criterio empleado por el Ministerio del Trabajo no consideró todas aquellas personas que, por muchas razones, renunciaron a la búsqueda de una ocupación.

EMPIRIA. Revista de Metodología de Ciencias Sociales. N. o 21, enero-junio, 2011, pp. 143-169. ISSN: 1139-5737 
Quedaron fuera los parados de la agricultura y los de las pequeñas empresas de carácter artesanal, pero sobre todo no se hacía referencia al fenómeno del subempleo meridional. La definición elegida por el ISTAT no convenció el secretario de la CGIL De Vittorio que requirió, sin éxito, a Barberi la creación de una comisión de estudio ampliada con una representación sindical. ${ }^{29}$ El ISTAT decidió no incluir en el censo general de la población del noviembre de 1951 la categoría «paro», a pesar de una explícita solicitud de Tremelloni en sede parlamentaria, fue en ese momento cuando se decidió el comienzo de la encuesta parlamentaria. ${ }^{30}$ Las cifras de la relación entre productividad y mercado del trabajo indicaron la necesidad de distinguir la realidad de Italia de la de Estados Unidos por la existencia de un paro de tipo estructural reabsorbible con el desarrollo industrial, y un paro no reabsorbible concentrado en las regiones rurales meridional; era necesario además, prever la posibilidad de un retorno masivo de emigrantes. La cuestión de la incongruencia de los datos provista por el ISTAT sólo fue afrontada al principio de los años sesenta. ${ }^{31}$

La cuestión del cálculo del número de parados se vinculaba a la definición de depresión meridional elaborada por Molinari. El concepto de Mezzogiorno había sido acuñado por la estadística del Risorgimento, pero sólo en la segunda posguerra, tras la invención de la contabilidad nacional fue utilizado como criterio de medición de la desigualdad de renta. ${ }^{32}$ Además del nivel de vida, tomó

${ }^{29}$ G. De Vittorio a B. Barberi. 22 de agosto 1951. Unos días después, siguió la respuesta de Barberi que contestaba amablemente pero sin prometer nada. B. Barberi a G. De Vittorio, 22 de agosto1951. Archivio histórico Istat, Relazioni con altre Pubbliche amministrazioni ed Enti, Varie, B. 3. Sobre Di Vittorio, véase la biografía de Craveri (1994).

${ }_{30}$ Barberi enseñó cierto escepticismo. Escribió a Tremelloni: «[...] Los resultados de la investigación serán más eficaces si se circusncriben al fenómeno del paro, resistiendo a la tentación de querer espaciar en campos ciertamente interesantes pero peligrosamente abiertos al subjetivismo de los entrevistadores o los relatores de la investigación». B. Barberi a R. Tremelloni, 14 enero 1952. Archivo histórico Istat. Relazioni con altre Pubbliche amministrazioni ed Enti, Varie, B. 3

${ }^{31}$ Las consideraciones de los más pesimistas hablaron de un paro de 3-4 millones de individuos. Fuentes oficiales se orientaron a acoger otras consideraciones pesimistas y en particular la cifra redonda de 2 millones de parados. En noviembre de 1955 el ISTAT instituyó un comité de estudio permanente sobre el fenómeno de la ocupación y el paro para contestar al Ministerio del Balance. A partir de segunda mitad de los años cincuenta, publicó una investigación trimestral sobre la fuerza de trabajo utilizando un criterio restrictivo, ya definido en las orientaciones angloamericanas. Emergía una profunda divergencia entre las cifras del Ministerio del Trabajo y las indicadas por el ISTATt. En diciembre de 1958 el jefe del gobierno Fanfani, de acuerdo con el ministro del Trabajo, Fiorentino Sullo, promovió la institución de una Comisión nacional para el estudio de las estadísticas del trabajo para superar las divergencias entre las dos fuentes oficiales. La Presidencia fue confiada al Ministro del Trabajo mientras que la Seguridad Social y la Vicepresidencia al Presidente del ISTAT, subrayando con eso la necesidad de colaboración entre el ministerio y el ISTAT. La Comisión entró en funcionamiento al principio de los Sesenta por iniciativa del ministro del Balance, Pella, y como soporte al inicio de la programacióon económica. Fue presidida por Giuseppe De Meo, presidente del ISTAT desde 1962.

${ }^{32}$ Estas dos nuevas subdivisiones estadísticas correspondían respectivamente a las regiones de Italia septentrional y central (Norte) y aquellas de Italia meridional e insular (Mezzogiorno, literalmente Mediodía)

EMPIRIA. Revista de Metodología de Ciencias Sociales. N. ${ }^{\circ}$ 21, enero-junio, 2011, pp. 143-169. ISSN: $1139-5737$ 
en consideración otros indicadores del bienestar, como el medioambiente, (el clima y la sismología), los servicios públicos (las infraestructuras, el crédito, el sistema de bienestar, la Caja para el Mezzogiorno, etc.) y el ámbito socio-cultural (la SVIMEZ 1954). ${ }^{33}$ La estadística sobre el Mezzogiorno iba más allá de la desigualdad de renta y recurrió a otros indicadores de desigualdad social (educación y medio ambiente). Este planteamiento fue presentada en un informe del Economic Commission for Europe, (ECE) de Ginebra sobre el desarrollo económico de los Países de la Europa mediterránea, pero no influyó en las políticas públicas. ${ }^{34}$ Este planteamiento se volvió a considerar y se desarrolló en los años ochenta por Amartya Sen y fu ratificada en los años noventa por las Naciones Unidas..$^{35}$

El punto de articulación en la historia de la renta nacional se encuentra en las discusiones alrededor del Esquema Vanoni, cuya elaboración se debe en buena medida al trabajo de la SVIMEZ entre 1953 y 1954 (Novacco 1996). A finales de 1953 el ISTAT llevó a cabo, por la primera vez, el cálculo agregado de la renta nacional en términos de producto (Carlucci Esposito, 2005) y publicó una serie histórica desde 1861, cuyo resultados confirmaban la tendencia del desarrollo italiano. ${ }^{36}$ Las imperfecciones del ISTAT fueron compensadas por otros importantes centros de encuesta de estadística económica, en primer lugar por el departamento de Estudios del Banco de Italia. ${ }^{37}$ Es en estos meses cuando se cumplen las primeras elaboraciones macroeconómicas para el cálculo de la coyuntura, según la

33 Fueron utilizadas centenares de publicaciones estadísticas, tanto oficiales como de estudiosos privados, aparecidas desde 1861 hasta 1953 sobre los censos, los catastros, los movimientos de la población, la instrucción pública, etc. Y aún, se utilizaron los resultados esenciales de estadísticas básicas ya acabadas, como por ejemplo la relativa a las empresas agrícolas censadas en 1930 o a la movilidad de los estudiantes superiores relativa al 1931.

${ }^{34}$ La iniciativa del ECE seguía al Survey ECE del 1953. Relación presentada a la XI Sesión del Consejo Económico y Social del ECE, en febrero de 1956, traducido y publicado en italiano por la SVIMEZ.

35 A Sen se le debe la formulación de una teoría de las elecciones sociales y la propuesta del Human Devevolpment Index (HDI) adoptado por las Naciones Unidas (Sen, 1992). En la última quincena se han hecho unas tentativas para llegar a una reforma del cálculo de la renta basado en el producto interior bruto. Acerca de la relevancia de las investigaciones de la SVIMEZ sobre la historia del pensamiento, véase Spalletti (2009).

${ }^{36}$ El ISTAT calculó los valores de la renta italiana al coste de los factores y al precio de mercado remontando hasta 1901. Los resultados se publicaron en Annuario Statistico de 1952. Las conclusiones fueron comentadas con cierto énfasis por el mismo Colin Clark (1954). En una siguiente publicación la colección histórica fue reconducida atrás, hasta el nacimiento de la Italia unida. Instituto Central de Estadística: «Investigación estadística sobre el desarrollo de la renta nacional de Italia de 1861 a 1956», en «Annali di statistica», a. 86, s. VIII, vol. 9, Roma, 1957. Sommario di statistiche storiche italiane 1861-1955, presentado por el ISTAT.

${ }^{37}$ Bankitalia con Salvatore Guidotti, en esa época dirigente y responsable del Servicio de Estudios desde 1956, lleva a cabo importantes estudios alrededor del problema de una política de programación. La contribución del Servicio de Estudios del Banco de Italia se tradujo principalmente en una aportación sobre el problema del cálculo de las disponibilidades financieras. R. De Bonis y A. Gigliobianco (2006)

EMPIRIA. Revista de Metodología de Ciencias Sociales. N. ${ }^{\circ}$ 21, enero-junio, 2011, pp. 143-169. ISSN: $1139-5737$ 
metodología programada por De Fenizio. El resultado de este trabajo fue la creación del ISCO, nacido el día siguiente de la formulación del Esquema Vanoni al que el gobierno confió la tarea de redactar la relación anual sobre la situación económica del país, comentando las estadísticas procedentes de las fuentes oficiales. La relación general de 1956 sobre la situación económica del país había sido perfeccionada respecto a las primeras versiones, como también Einaudi reconoció, aunque entrevió la permanencia del defecto de «soberbia estadística» que obligaba a los lectores a descifrar las palabras «cultas» utilizadas en la relación. «Me corto la lengua si, entre los 800 y más parlamentarios, a quienes, en primer lugar, está destinado el informe, más del 5 por ciento es capaz de entender bien lo que está escrito en el volumen. Todo el mundo simula entender; pero es pura simulación». ${ }^{38}$ Para los periodistas, publicistas y economistas la cifra del 5 por ciento era generosa en relación al concepto de valor añadido inventado por los estadístico y utilizado por el ISTAT. Actitud que denotaba un desprecio de los compiladores hacia los lectores. Einaudi volvía a acusar, como ya lo había hecho anteriormente, de que en la valoración de la renta de los edificios no se tenía en cuenta que los alquileres estaban bloqueados. La falta más grave se comprobaba en el criterio empleado para el cálculo del «producto neto de la administración pública con resultados completamente inverosímiles». ${ }^{39}$

\section{LAS CUENTAS NO CUADRAN}

A lo largo de los años sesenta también Italia se dotó con un instrumento de contabilidad nacional adecuado para enfrentar los problemas de regulación del crecimiento. En esta resolución se influyeron tres factores: el cambio de la coyuntura económica internacional, cuyos efectos se hicieron sentir en el curso de la década; el inicio del proceso de integración europea y también el cambio de la política interna, con el nacimiento del centroizquierda y la entrada del Partido Socialista en el gobierno. En este período, empieza a operar el Development Center de la Organisation de Coopération et de Developpment Économiques (OCDE) de París, vinculada al Banco mundial..$^{40}$ En 1961 la Comunidad Económica Europea, decidió la creación de un centro de encuesta estadística (Eurostat) y este acontecimiento aceleró la solución del problema del cálculo de la conta-

${ }^{38}$ L. Einaudi a A. Zoli, ministro del Bilancio, 2 maggio 1957. ASBI, pratt., n. 288, fasc. 9.

${ }_{39}$ El producto neto de la administración pública, escribió con ironía, era «lo que de nuevo crean, arrancan del nada los funcionarios públicos, el producto nuevo que extraen de su propia cabeza y de su propia fatiga, como lo hace el campesino con el trigo, es medido por los sueldos y cheques que ellos cobran». Si esta sentencia hubiera sido expresada con palabras simples habría sido acogida por una sonora carcajada.

40 El economista Angus Maddison ha llevado hasta sus extremas consecuencias, las potencialidades analíticas del método de la estadística histórica para analizar los niveles de diferencia en la historia mundial entre las naciones ricas y pobres (Maddison 1992; 2008).

EMPIRIA. Revista de Metodología de Ciencias Sociales. N. ${ }^{\circ}$ 21, enero-junio, 2011, pp. 143-169. ISSN: $1139-5737$ 
bilidad nacional. En 1962 se publicó una colección histórica sobre el crecimiento en el sexenio 1953-59. Además, al censo de la población de 1961 se añadió la tercera consulta sobre la industria y el comercio y también una encuesta estadística de la agricultura que iba a unirse al sondeo mundial promovido por la FAO. En realidad, los nuevos censos del ISTAT no se diferenciaban mucho del planteamiento de 1951-53, y en tal circunstancia volvieron a considerarse las líneas de investigación promovidas por la Comisión Constituyente: el estudio sobre el fenómeno de la concentración industrial y social y sobre la diferencia regional. Ambas reformas se fijaron en la perspectiva de favorecer una cultura del mercado.

Los grupos de centroizquierda, antes que sancionar el reconocimiento del reformismo liberal, promovieron el principio de un nuevo curso de la política económica con resultados fuertemente contradictorios. Prevaleció la consigna antiliberal de las reformas de estructuras y la programación, entendidas como instrumento de transformación general del sistema. Es elocuente la escasa atención manifestada por las fuerzas de la mayoría hacia la iniciativa de la Comisión de Investigación sobre los límites de la competencia ${ }^{41} \mathrm{y}$, por otro lado, la quiebra del proyecto de La Malfa, ministro de Hacienda, para aplicar a Italia la política de rentas, ya experimentada con éxito en Gran Bretaña. En 1963-64 se abrió un conflicto entre el partido de los inflacionistas y los defensores de la estabilidad monetaria, testimoniando la debilidad en la capacidad de gobernar la economía. La crisis fue solucionada por la intervención sustitutoria del gobernador de Bankitalia, Guido Carli, quien puso en discusión el poder de las instituciones parlamentarias. En esta coyuntura emergió la personalidad de Baffi, Director General del Banco de Italia, que vio en la consolidación de los instrumentos de análisis económico y financiero la solución del problema. El verdadero peligro, le escribió a la economista Vera Lutz, que había tomado una posición de crítica liberal, «no se encontraba en las erradas administraciones regionales y sectoriales de inversión, sino en las ulteriores nacionalizaciones...». ${ }^{42}$ Baffi adoptó una actitud dialogante con el nuevo gobierno, haciendo presión sobre la relación directa con La Malfa. Pocos días después, cuando La Malfa fue nombrado ministro del Balance, Baffi le habló de sus predecesores Einaudi y Vanoni, y lo invitó a asumir el papel de «elder statesman» de la política económica italiana. ${ }^{43} \mathrm{Com}-$

\footnotetext{
${ }^{41}$ Eso es lo que emerge en su relación sobre 16 actividades productivas, presentada a la Comisión. (Battara, 1963b; 1965). En realidad, el gobierno de centroizquierda orientó la acción a favor de la intervención del Estado en la economía. Por una reconstrucción histórica, (véase: Granata 2008).

${ }^{42} \mathrm{Y}$ continuó «Mi gran rabia es que esta nueva política sea el resultado de un grupo predominantemente milanés con los que tengo familiaridad de treinta años: Tremelloni, La Malfa, Fanfani, Sarraceno, Parri...¿Quos Deus vult perder dementat? Quizás el señor quiera perder el Val Padana: ¿pero por qué, si es allí donde encuentra a los últimos cristianos?». P. Baffi a V. Lutz, 23 febrero 1962. ASBI, Carte Baffi, Monte Oppio, Pratiche, Corda 226.0, F. 13.

${ }^{43}$ Baffi a Ugo La Malfa, 22 de febrero1962. La Malfa contestó de manera sibilina: «Querido Baffi, has hecho bien al recordarme [...] Einaudi y Vanoni. En su recuerdo, trataré de hacer cuanto pueda para la economía de nuestro País sin...decepcionarte». U. La Malfa a P. Baffi, 26 de febrero de 1962.
}

EMPIRIA. Revista de Metodología de Ciencias Sociales. N. ${ }^{\circ}$ 21, enero-junio, 2011, pp. 143-169. ISSN: 1139-5737 
partió la posición de firmeza asumida por el ministro del Balance relativa a la solicitud de control sindical en la dirección de las empresas públicas (ASBI 1962,Carte Baffi, fasc. «La Malfa, Ugo»). En otra carta, escrita después de cuatro años, le aconsejó a La Malfa que renunciara a la perspectiva de aplicación de la política de las rentas bien porque en Italia, a diferencia de lo que ocurría en Gran Bretaña, los efectos previstos no se podrían alcanzar por la falta de colaboración por parte de Confindustria y del sindicato, bien porque el curso de la coyuntura dejó pocos márgenes al relanzamiento de esta estrategia. En otras palabras consideró inadecuada la política de las rentas para hacer frente a la coyuntura caracterizada por una estabilidad monetaria. ${ }^{44}$ De tal hecho emergía la escasa independencia de la información estadística que tenía que orientar las decisiónes del gobierno. En esta circunstancia, el Banco de Italia promovió la creación de una comisión de estudio en la que también trabajó el economista Franco Modigliani, actualizando el criterio de construcción de la contabilidad nacional. Se estableció una relación explícita entre inversiones y su sostenibilidad para enfrentar el crecimiento del déficit público y la inestabilidad monetaria. Con esta iniciativa, Italia emprendió una revisión crítica en sentido liberal del pensamiento de Keynes. (Masera, Fazio Padoa-Schioppa 1975; Baffi 1990, 139-145; Sylos-Labini 2006). La crisis de 1963-64 había puesto en evidencia el problema de la necesidad de una mayor cautela en el empleo de las cifras. (Battara, 1963 237-238). La consolidación del marco conceptual de la contabilidad nacional era un instrumento indispensable para circunscribir la aplicación de una política de déficit, se había alcanzado el objetivo de un conocimiento general y exhaustivo de las fuerzas económicas y sociales, dando un vuelco a la crítica liberal einaudiana en sentido reformista: el problema era político. El socialismo italiano habría tenido que renunciar al mito de las reformas estructurales y volver a la concreción de la realidad y ponerse como objetivo el de garantizar la libertad de elección económica de a los ciudadanos. Con la crisis del petróleo y el crecimiento de la inflación de los años setenta, se descubrió que la dirección de gobierno había preferido la negociación entre intereses particulares y clientelares a la política de reformas.

${ }^{44}$ Escribió una carta a comentario del artículo de La Malfa, Unanimità non programmatoria publicado por «La Voce Repubblicana», en la que - polemizando con Amendola y Giolitti - La Malfa declaró que la política de rentas era un factor decisivo para la suerte de la izquierda en Europa. Por otro lado, Baffi le escribió a La Malfa que la política de rentas había sido sobrestimada. En Gran Bretaña ocasionó una pérdida de terreno tanto en la producción como en los cambios internacionales. Podía ser útil en Italia solamente como correctivo de la dinámica de crecimiento, para combatir las zonas de privilegio corporativo. Escribe Baffi: «Para este objetivo, la política de rentas tiene que procurar, junto al objetivo de la contención de la demanda monetaria global, la reducción de los grupos protegidos al nivel mediano: empezando por sector público entendido en sentido amplio. Por esto, el discurso debería centrarse sobre todo en la relación remuneración/productividad, sin una insistencia exclusiva sobre la primera variable, o sea sobre el hecho monetario con respecto de lo real». P. Baffi a U. La Malfa, 3 agosto 1966. ASBI, Carte Baffi, fasc. «La Malfa, Ugo».

EMPIRIA. Revista de Metodología de Ciencias Sociales. N. ${ }^{\circ}$ 21, enero-junio, 2011, pp. 143-169. ISSN: $1139-5737$ 
En conclusión, se pueden hacer dos consideraciones de carácter general. Italia participa en la historia internacional de la contabilidad nacional, y parece incapaz de romper el vínculo ejercido por el peso de las ideologías partidarias. Este factor se configura en los años treinta y se impone, al término de la primacía de los partidos políticos, en los años sesenta con el nacimiento de la sociedad de consumo de masas. Consecuencia de todo ello es la reducción de los espacios de autonomía de la estadística pública respecto a los intereses corporativos y la formación de una élite tendencialmente tecnocrática, generadora de nuevas ilusiones pero funcional a los intereses de los partidos. Un indicio de ello se encuentra en el curso, contado en este estudio, de la cultura liberal y reformista que se abre al lenguaje matemático a partir de 1900 en continuidad con la corriente de las encuestas económicas y sociales. En este contexto cultural se inaugura un «nuevo reformismo», nace un intelectual no político, pragmático que rompe con el conformismo. Estos autores han propuesto análisis e interpretaciones que ilustran los problemas típicos de una sociedad industrial madura, aunque no han logrado incidir en la decisión política. Han comprendido con antelación los cambios de la economía mundial, aunque han sido excluidos del gobierno de la economía. La nueva crisis económica surgida en 2007, hace actual el discurso einaudiano sobre la necesidad de un conocimiento científico correcto y sobre las dificultades de una previsión del futuro por la falta de datos o de cifras probablemente falsas. La crítica liberal contra la presunción de los economistas se traduce hoy en la batalla en defensa de la libertad de crítica y de una información independiente. Quizás sea este el principal y más duradero legado del reformismo liberal.

\section{BIBLIOGRAFÍA}

BAFFI P. (1990): Testimonianze e ricordi, Mián, Libri Scheiwiller.

BAFFIGI A. (2007): «Cultura statistica e cultura politica: 1'Italia nei primi decenni unitari», Quaderni dell'Ufficio Ricerche Storiche, n. 15, mayo.

Banca d'Italia (2006): «I conti finanziari: la storia, i metodi, l'Italia e confronti internazionali», Atti del Convegno, Perugia, 1-2 dicembre.

BARBERI B. (1947): «Alcune considerazioni sul concetto di reddito nazionale», L'Industria, n. 4.

BARBERI B. (1948): «Alcune considerazioni sulla costruzione dei numeri indici», Annali di statistica, s. 8, vol. 2.

BARBERI B (1949): Il reddito nazionale e la bilancia dei pagamenti: elementi definitori e schemi rappresentativi, Roma, Failli.

BARUCCI P. (2008): Mezzogiorno e intermediazione «impropria», Bologna, Il Mulino.

BATTARA P (1950): «Contabilità economica nazionale delle transazioni», Moneta e credito, v. III, n. 3, pp. 339-362.

BATTARA P (1955): «Esame critico della definizione corrente di reddito nazionale», Economia Internazionale, v. 8, n. 3, pp. 457-502.

BatTARA P. (1963a): «Luci ed ombre nel calcolo del reddito nazionale», Moneta e Credito, v. XVI, n 2.: 193-239.

EMPIRIA. Revista de Metodología de Ciencias Sociales. N. o 21, enero-junio, 2011, pp. 143-169. ISSN: 1139-5737 
BATTARA P. (1963b): «Ricerche induttive sulla libertà di accesso delle imprese nel mercato», en Saraceno P. e Tagliacarne G. (eds.), pp. 93-130.

BATTARA P. (1965): «La concentrazione industriale», en Atti della Commissione parlamentare di inchiesta sui limiti posti alla concorrenza nel campo economico, t. 4 Roma, Camera dei deputati, Servizio studi legislazione e inchieste parlamentari.

BEAUD J. - Prévost J. G. (eds) (2000): L'ère du chiffre: systèmes statistiques et traditions nationals / The Age of Numbers: Statistical Systems and National Traditions, Montréal, Press de l'Université du Québec.

BetTelheim C. (1949): La pianificazione sovietica, Mián, Edizioni di Comunità.

Bini P. E MazziotTA C. (eds.): (2004): Sviluppo economico e istituzioni: la prospettiva storica e l'attualità: scritti in memoria di Massimo Finora, Mián, Angeli.

CARluCCI M. E Esposito G. (eds.) (2005): Statistica economica e strumenti di analisi: studi in memoria di Antonino Giannone, Roma, IS.CO.NA.

CLARK C (1954): «Lo sviluppo dell'economia italiana: l'incremento del reddito reale per uomo-ora dal 1901 al 1953», en Moneta e Credito, vol. II, no. 3, pp. 261-264.

Cotula, E. Gelsomino C. O. y GIGLIOBIANCO A. (1997): (eds):, Donato Menichella. Stabilità e sviluppo dell' economia italiana 1946-1960, 2. vol, Roma-Bari.

CRAVERI P. (1994): «Di Vittorio Giuseppe», en Dizionario biografico degli italiani, Istituto della Enciclopedia Italiana, Roma, Ad vocem.

De Bonis R. E Gigliobianco A. (2006): «Le origini dei conti finanziari negli Stati Uniti e in Italia: Capeland, Baffi, le istituzioni», in I conti finanziari, cit. pp. 31-32.

DESRORIÈSES A. (1993): La politique des grands nombres: histoire de la raion statistique, La Découverte, Paris.

DESRORIÈSES A (2000): Histoire de la statistique: styles d'écriture et usages sociaux, in Beaud e Prévost: 37-57.

DIDIER E. (2009): L'Amerique dans les chiffres. Le New Deal et l'émergence des statistiques pubbliques aux E'tats-Units, La Dòcouverte, 2009.

Di FENIZIO F. (1950): «Dalla "Relazione generale 1949" alla "Relazione generale 1950"», in L'Industria, n. 3: 347-389.

EINAUIDI, L «Discorso all'Assemblea Costituente del 18 giugno 1947», en Atti parlamentari dell'Assemblea Costituente, pp. 4963 e 4965.

FAVEro G. (2001): Le misure del Regno. Direzione di statistica e municipi nell'Italia liberale, Padua, Il Poligrafo.

FAVERo G (2009): «Gli statistici e la statistica nell'Italia del Novecento», in Storia del pensiero economico, a. 6, n. 1, pp. 157-166.

GERBI S (2002): Raffaele Mattioli e il filosofo domato, Turín, Einaudi.

Gigliobianco A. (2006): Via Nazionale. Banca d'Italia e classe dirigente; cento anni di storia, Roma, Donzelli.

GUARINI R. (2003): «Le misurazioni dell'attività economica», in La storia della statistica pubblica in Italia, Livia Linda Rondini (ed):, Mián, Angeli, pp. 39-53.

GranATA, M. (2008): Cultura del mercato. La Commissione parlamentare d'inchiesta sulla concorrenza (1961-1965):, Soveria Mannelli, Rubbettino.

ISTAT (1950): Censimento industriale e commerciale 1937-1940, Roma, Failli.

LENTI L. (1943): «Primi risultati del censimento italiano della produzione 1937-1938», «Rivista bancaria delle assicurazioni e dei servizi amministrativi, enero, pp. 15-30.

LENTI L. (1983): Le radici nel tempo. Passato al presente e futuro, Milán, Angeli.

MADDison A. (1992): «L'économie mondiale 1820-1992», Analyse et statistiques, Paris, OCDE 
MAdDISOn A. (2003): The world economy: historical statistics, Paris, OCDE.

MARUCCO D. (1996): L'amministrazione della statistica nell'Italia unita, Bari, Laterza.

MASERA F. FAZIO A. PADOA-SchiopPA T. (1975): Econometric research in European Central Banks, Roma, Banca d'Italia.

Meynell, A., (1960): La politica inglese di localizzazione dell'industria (1934-1959):, Roma, Giuffrè Editore

Misiani S (2007): I numeri e la politica. Statistica, programmazione e Mezzogiorno nell'impegno di Alessandro Molinari, Bologna, Il Mulino.

Molinari A. (1937): Istituto centrale di statistica nel 1936. Relazione al Consiglio superiore di statistica nella seduta del 21 dicembre 1936, Roma, Istituto Poligrafico dello Stato.

MOLINARI A. (1942): Statistica delle macchine generatrici di energia e calcolo della potenza utilizzabile, Roma, Tip. Fausto Failli.

MOLINARI A. (1947): La statistique internationale des forces motrices, Rapporto alla XXV Sessione dell'Istituto internazionale di statistica, 6-18 settembre, Washington.

NALDI, NERIO, (2000): «The friendship between Piero Sraffa and Antonio Gramsci in the years 1919-1927», in European Journal of history of economic thought, vol. 7 n. 1 , pp. 79-114.

Novacco N. (1996): Politiche per lo sviluppo. Alcuni ricordi sugli anni '50 tra cronaca e storia, Boloña, Il Mulino.

OECE (1952):, A standardised system of National Accounts, OECE.

REY G. (ed.) (1991): I conti economici dell'Italia, Roma-Bari, Laterza.

Rodano, GIULIO (1983): Il credito all'economia. Raffaele Mattioli alla Banca Commerciale Italiana, Ricciardi, Miáno-Napoli.

RONDINI L. L. (ed.) (2003): La storia della statistica pubblica in Italia, Mián, Angeli.

SARACENo P. E TAgLIACARNE G. (eds.) (1963): Scritti di economia e statistica in memoria di Alessandro Molinari, a cura di Miáno, Giuffrè.

SEN A (1992): Inequality reexamined, Oxford, Clarendon.

SYlos LABINI P. (2006): Torniamo ai classici: produttività di lavoro, progresso tecnico e sviluppo economico, Roma-Bari, Laterza.

SPALlETti S. (2009): Istruzione, crescita e rendimenti nella teoria del capitale umano. Una prospettiva di storia del pensiero economico, Roma, Aracne.

Svimez (1954): Statistiche sul Mezzogiorno d'Italia, 1861-1953, Roma.

SVIMEZ (1961): Un secolo di statistiche italiane. Nord e Sud, 1861-1961, Roma.

TUCCIMEI E. (2005): «La ricerca economica a Via Nazionale. Una storia degli "Studi” da Canovai a Baffi (1894-1940):», Quaderno dell'Ufficio Ricerche Storiche, n. 9, septiembre.

VANOLI A. (2005): A history of nazional accaunting, Amsterdam, IOS Press.

\section{RESUMEN}

El artículo ofrece una breve introducción a la historia de la contabilidad en Italia y examina, en particular, los orígenes de la decisión política. Esto ensayo analiza la posición preeminente, aunque largamente ignorada, ocupada en el pensamiento empírico por parte del debate público sobre la renta pública. La con-

EMPIRIA. Revista de Metodología de Ciencias Sociales. N. ${ }^{\circ}$ 21, enero-junio, 2011, pp. 143-169. ISSN: 1139-5737 
tabilidad nacional se ha planificado en Italia sin dar lugar a una democracia liberal, como fue el caso de los Estados Unidos. El instituto nacional de estadística italiano (ISTAT) fue controlado directamente por el fascismo. Mussolini usaba la estadística como un instrumento para el control totalitario. Pero una análisis más profundo de esta institución contradice esta afirmación. Se abre una contradicción entre la imagen de propaganda del fascismo y las mediciones hechas por el ISTAT. Hoy, después de la crisis económica y financiera mundial del 20072009, la historia de la contabilidad italiana es aún un tema de actualidad.

\title{
PALABRAS CLAVE
}

Molinari, Einaudi, contabilidad nacional, planificación económica, mezzogiorno, ISTAT, Svimez

\begin{abstract}
The article offers a short introduction to the Italian accounting history and discusses, particularly, on the origins of political decision. This essay analyses the preeminent, but largely ignored, position occupied by the empirical statistical method in public discussion of the Italian income. The national accounting was planed in Italy without giving way to a liberal democracy, as it the case in United States. Istat was directly controlled by Fascism. Mussolini used statistics as a tool for totalitarian control. But closer analysis of the story of this institution contradicts this assumption. There is a contradiction between the propaganda image of Fascism and the Istat direction line. Even in the present, after the Global Financial Economic Crisis 2007-2009, the Italian accounting history is still topical.
\end{abstract}

\section{KEYWORDS}

Molinari; Einaudi; national accounts; economic planning; Mezzogiorno; ISTAT; Svimez 